\title{
A PATIENT WITH INTERMEDIATE SYNDROME WITH TOXIC ENCEPHALOPATHY FOLLOWING ORGANOPHOSPHATE POISONING
}

\author{
MOHIUDDIN SHARIF ${ }^{1}$, MUKTADIR BHUIYAN $^{2}$, RAJIB ROY $^{1}$, MAHFUZUL HAQUE $^{3}$, MD ROBED AMIN $^{4}$
}

\begin{abstract}
:
OP poisoning is the common cause of mortality and morbidity in Bangladesh. Although acute cholinergic crisis is the common form of presentation of such cases, the intermediate syndrome and delayed encephalopathy can be also presented in op compound. A 14 year old adolescent presented with acute onset of intermediate syndrome and simultaneous early encephalopathy after ingestion of combination of Chlorpyrifos with Cypermithrin known popularly as market name of 'Mortar'. The patient was provided supportive care and few days of ICU care with complete recovery. The interesting feature of the case was absence of cholinergic crisis and hence patient was not treated with atropine or pralidoxime.The simultaneous combination of presenting features, prolonged coma and even on absence of specific antidote complete recovery was the highlighting points of this unique case.
\end{abstract}

Keywords: OP compound, Intermediate syndrome, Encephalopathy, Chlorpyriphos

Received: 16 September 2018

Accepted: 05 November 2018

DOI: https://doi.org/10.3329/bjmed.v30i1.39923

\section{Introduction:}

Organophosphates (OP) are primarily used as pesticides and suicidal ingestion, occupational exposure, or consumption of oil or food contaminated by organophosphates is well documented modes of poisoning. The severity of the poisoning depends on the dose and the type of organophosphate exposure. Commonly the fat soluble OP compound presented with acute cholinergic crisis with features of respiratory failure as severity ${ }^{1}$. OP compound can also presented with intermediate syndrome and Organophopshate compound related delayed polyneuropathy (OPIDP) ${ }^{1,2}$ Although there has been cases where the toxic encephalopathy was featured in OP compound, the common form of which was posterior reversible encephalopathic syndrome (PRESS). Several clinical syndromes can be encountered in OP but intermediate syndrome with toxic encephalopathy followed by organophosphate poisoning (Chlorpyrifos with Cypermithren) has not been found commonly in toxciology. Here is a case report of such entity with better outcome.

\section{Case Summary:}

A 14 year old girl presented to the Dhaka Medical College Hospital (DMCH) in Bangladesh complaining about weaknesses of lower limbs and dropping of upper eyelids of both eyes. Upon further enquiry, the patient revealed the consumption of organophosphate compound (Chlorpyrifos with Cypermithren) intended to commit suicide 6 hours ago after taking approximately $20 \mathrm{~mL}$ of combined product with the trade name of 'Mortar'. She did not receive any treatment outside the Hospital.

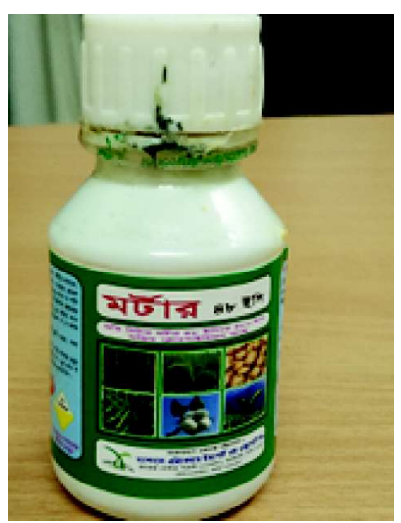

Fig.-1: The brought specimen showed OP compound Morter (Chlorpyriphos and Cypermethrin)

1. Medical Officer, Department of Medicine, Dhaka Medical College

2. Assistant Registrar, Department of Medicine, Dhaka Medical College

3. Consultant of Medicine, Department of Medicine, Dhaka Medical College

4. Associate Professor of Medicine, Department of Medicine, Dhaka Medical College

Address of Correspondence: Dr.Md Robed Amin, Associate Professor of Medicine, Dhaka Medical College. Room no-502, Department of Medicine, Dhaka Medical College and Hospital-2. Email-robedamin@yahoo.com 

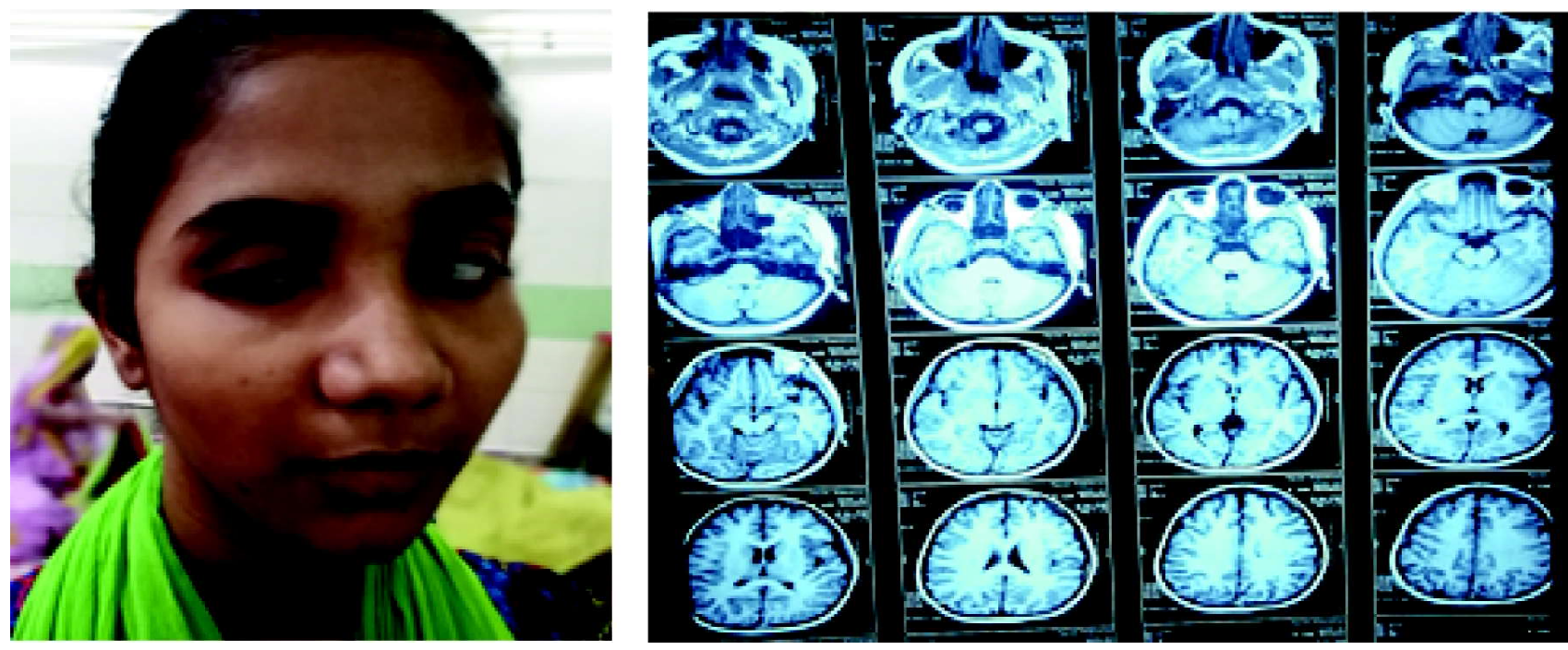

Fig.-2 (a\&tb): Bilateral ptosis and mild external opthalmoplegia and MRI revealed hyperintensity in temporal lobe

On admission, she was drowsy with mild respiratory distress. There was nausea but no vomiting or diarrhea. The pupils were mid-dilated reacting to light. The pulse was $110 \mathrm{bpm}$ and blood pressure 100/60 mmHg. Lungs were clear. No obvious salivation, lacrimation or fasciculation was observed.

Laboratory examinations revealed a white blood cell count of 13,780/mL, sodium $136 \mathrm{mEq} / \mathrm{L}$, potassium $4.0 \mathrm{mEq} / \mathrm{L}$ and creatinine $0.69 \mathrm{mg} / \mathrm{dL}$. SGPT $13 \mathrm{u} / \mathrm{L}$, SGOT $25 \mathrm{u} / \mathrm{L}$ and S.bilirubin $0.65 \mathrm{mg} / \mathrm{dL}$. An electrocardiogram showed normal sinus rhythm.

After an hour of admission, we noticed that her condition was deteriorating and there is an increase in respiratory rate, sweating, restlessness and later cyanosis. Therefore, she was transfer to the ICU where she remained for subsequent 4 days. In ICU, she was put on assisted respiratory support with high flow oxygen but no intubation was done. They were not sure about the agent responsible for such dramatic respiratory distress and so treated her with antibiotics, supportive care with nebulization and constant oxygen delivery. Her Glasgow coma score was below 9 with low systolic blood pressure which responded to intravenous saline. During her stay in ICU for 96 hour, there was slow improvement happens on vital signs and gradual stability in respiratory effort. As her condition improves except the semi-unconsciousness, she was returned to ward. At the ward, we noticed that her vital signs have become stable but she was still unconscious. Then, an MRI of her Brain was done and it was found unremarkable. She regained her consciousness 2 days later and neurological examination was consistent with that weaknesses of both of her upper and lower limbs and ptosis of both upper eyelids. Her consciousness return to completely normal state and she could not remember any event of prolonged unresponsiveness. A nerve conduction study (NCS) of Cross Limbs and CSF Study was done which were also unremarkable. We carried out some investigation and the report showed that on investigation, Complete Blood Count (CBC), blood sugar, LFT, KFT, serum electrolytes, ECG, and X-ray chest were normal. Upon continued observation, we noticed that at around Day 17 of her stay in $\mathrm{DMCH}$, her weakness started to improve and she could move her limbs and sit upwards on the bed. During her arrival the patient did not receive any atropine and pralidoxime as an antidote as classical acute cholinergic crisis was not present. Her respiratory distress as recovered with high flow oxygen and occasional nebulae. Her weakness and proximal following distal weakness was only observed and only physiotherapy was advocated.

\section{Discussion:}

Organophosphate compounds (OPCs) are used as pesticides, petroleum additives, plastic modifiers, lubricants, antioxidants, and flame retardants. They may be absorbed via the skin or respiratory and gastrointestinal tracts. Suicidal ingestion is the most common mode of OPC poisoning in India. Occupational exposure to organophosphates in agricultural workers or consumption of oil or food contaminated or adulterated by the toxin are other well documented modes of poisoning. The severity of poisoning depends on the dose and the type organophosphate exposure. 3 Commonly implicated OPCs include diazinon, malathion, fenthion, sumithion, chlorpyrifos, chlorfenvinphos, paroxanmethyl, dimethoate, oxydementon, tri-orthocresyl phosphate (TOCP), dichlorovos, leptophos, mipafox, chlorphos, trichlorfox, metriphonate and metamidophos. 3,4

The three phases of neurological illness following OP intoxication are well reported. Phase I (acute cholinergic crisis) occurs predominantly quickly after ingestions 
and it is the common presentation and physicians dealt this presentation with atropine and pralidoxime. Phase II (intermediate syndrome) develops 24-96 hours during and sometimes after resolution of $1^{\text {st }}$ phase. It is characterized by ptosis, opthalmoplegia, broken neck signs and also weakness of proximal muscles of specially limbs, and cranial nerve palsies. Extrapyramidal symptoms are uncommon, but can be a serious occurrence in OP poisoning in Phase II. 5 Phase III (organophosphate-induced delayed polyneuropathy) presents 2-3 weeks after OP exposure and predominantly motor in type. ${ }^{6}$

The intermediate syndrome has variable presentation and variable severity with mild proximal weakness to severe type II respiratory failure and in need of mechanical ventilation. ${ }^{5,6}$ In out index case, the patient developed ptosis and opthalmoplegia and having impending respiratory failure. As she was referred to ICU for special support, her progression gradually stopped and her respiratory drive gradually turn back to normal with only oxygen therapy and occasional nebules. The severity of respiratory distress and quicker non progression was a strange finding in this case and it provokes more basic works on intermediate syndrome. In our case the patient had steady improvement on the part of intermediate syndrome and follow up after one month showed complete recovery from intermediate syndrome.

The interesting finding of this index case was her state of encephalopathy. Although PRESS has been described in few literatures but commonly it is associated with characteristic imaging findings in CT and MRI. The delayed type of encephalopathy was described 35 patients in one original study ${ }^{7}$ where the GCS was very low after about 5 days with miosis with clinically detectable no cortical or brainstem activity and computed tomography of the brain and cerebrospinal fluid analysis were normal and Electroencephalogram showed bihemispheric slow wave disturbances. In our index case, the interesting finding was early encephalopathy within 6 hours of time and persisting for prolonged duration of a week. The ingredients of cypermethrin (high percentage in combination with chlorphyrifos) may be also responsible for such early encephalopathy but the presentation of intermediate syndrome at same time make the chlorphyrifos as the likely culprit agent for early encephalopathy. The imaging study in the index case was unremarkable with mild hyperintensity noted in temporal zone and this is consistent with prior study. ${ }^{4,7}$ Although persistent cholinesterase inhibition is likely to have contributed to the manifestations, the mechanism of coma and encephalopathy need to be explored in further trials. The good outcomes in this patient suggest that therapy should not be limited in OP-poisoned patient developing profound coma or encephalopathy during hospitalization. Also, we followed up with her condition after one month and found that she has now recovered from the symptoms of toxic encephalopathy completely.

In patients who are presenting with intermediate syndrome and toxic encephalopathy following exposure to organophosphates, particularly chlorpyrifos, should be kept under observation. Some patients with intermediate syndromes might get better gradually without any specific treatments and further follow-up of patients suffering from organophosphate poisoning are also necessary to prevent the development of further complications such chronic organophosphate poisoning induced neuropathy.

\section{Conclusion:}

Understanding that the OP intermediate syndrome with the neurological complications of encephalopathy may complicate the diagnosis and treatment of OP intoxication indicate discussion among medical students, medicine residents, emergency residents, and primary care physicians in rural practice where OP intoxication cases may be anticipated. The primary protective measure to avoid both OP intoxication is to establish national policies, comprehensive regulations, and education for the safe handling and storage of OPs for domestic, agriculture, and industrial use.

\section{References:}

1. National guideline of management of poisoning in Bangladesh. 2007.DGHS,Bangladesh

2. Center for Disease Control. Organophosphorus insecticides: Dialkyl phosphate metabolites. National Report on Human Exposure to Environmental Chemicals-Fact Sheet. 2011. [Last accessed on 2014 Jan 17]. Available from: http:/ /www.cdc.gov/exposure report/OP.DPM_FactSheet.html.

3. Singh S, Sharma N. Neurological syndromes following organophosphate poisoning. Neurol India. 2000;48: 308-13. [PubMed]

4. Hsieh BH, Deng JF, Ger J, Tsai WJ. Acetylcholinesterase inhibition and the extrapyramidal syndrome: A review of the neurotoxicity of organophosphate. Neurotoxicology. 2001;22:423-7. [PubMed]

5. Senanayake N, Sanmuganathan PS. Extrapyramidal manifestations complicating organophosphorous insecticide poisoning. Hum Exp Toxicol. 1995; 14:6004. [PubMed]

6. Tanima D, Maisnam I, Kundu AK, Saha SP, Ghosh S, Maity A. Atypical central nervous system involvement in acute organophosphorus poisoning. J Assoc Physicians India. 2011;59:326-7. [PubMed]

7. Peter JV, Prabhakar AT, Pichamuthu K. Delayed-onset encephalopathy and coma in acute organophosphate poisoning in humans. Neurotoxicology. 2008 Mar;29(2):335-42. doi: 10.1016/j.neuro.2008.01.004. Epub 2008 Jan 21. 